\title{
O conhecimento de pediatras sobre alergia alimentar: estudo piloto
}

\author{
Pediatricians' knowledge on food allergy: pilot study
}

Dirceu Sole 1 , Cristina Miuki A. Jacob², Antônio Carlos Pastorino², Arnaldo Porto Neto ${ }^{3}$, Dennis Alexander Burns ${ }^{4}$, Emanuel Sávio C. Sarinho ${ }^{5}$, Evandro Alves do Prado ${ }^{6}$, Francisco José P. Soares ${ }^{7}$, Maria Cândida V. Rizzo ${ }^{8}$, Maria das Graças N. Silva ${ }^{9}$, Norma de Paula Rubini ${ }^{10}$

\section{RESUMO}

Objetivo: Avaliar o conhecimento de pediatras sobre alergia alimentar.

Métodos: Dados obtidos de questionário padronizado, postado e respondido por pediatras filiados à Sociedade Brasileira de Pediatria (SBP). Digitação dos dados em planilha Excel e análise de freqüência de respostas afirmativas em porcentagem.

Resultados: Foram analisados 895 questionários preenchidos por pediatras de todo o país, com predomínio da região Sudeste $(61,6 \%)$. Segundo os pediatras entrevistados, as manifestações diagnósticas de alergia alimentar são: respiratórias, cutâneas e sistêmicas, em iguais proporções. Ainda segundo estes pediatras, leite de vaca $(98,9 \%)$, clara de ovo $(58,7 \%)$ e amendoim $(50,9 \%)$ são os principais alimentos associados a essas manifestações. Embora 74,8\% dos respondedores tivessem identificados os corantes e aditivos alimentares como responsáveis pela alergia alimentar, apenas 19,4\% conheciam o código de identificação da tartrazina.

Conclusões: Os dados apresentados reforçam a necessidade de ampliação dos conhecimentos dos profissionais de saúde sobre o diagnóstico e tratamento da alergia alimentar, com o objetivo de garantir o uso de critérios diagnósticos e terapêuticos mais adequados.

Palavras-chave: hipersensibilidade alimentar; conhecimento; criança; aditivos alimentares; alimentos; substitutos do leite; clara de ovo; amendoim; tartrazina.

\section{ABSTRACT}

Objective: To evaluate the knowledge of Brazilian pediatricians about food allergy.

Methods: Data was obtained from a sent back posted written questionnaire. It was filled in by Brazilian pediatricians, affiliated to the Brazilian Society of Pediatrics. Data was transcript to an Excel spread sheet and the frequency of affirmative responses was reported as percentages.

Results: Data from 895 written questionnaire of pediatricians from all over Brazil, (mainly from the southeastern region $-61.6 \%)$, were analyzed. The main clinical expressions of food allergy determined by the pediatricians were: respiratory, cutaneous and systemic symptoms (equal proportions). According to these pediatricians, cow's milk (98.9\%), egg white (58.7\%) and peanuts $(50.9 \%)$ were the main allergens related to food allergy symptoms. Although $74.8 \%$ of the responders have indicated food dyes and food additives as associated to food allergies, only $19.4 \%$ of them knew the identification code of tartrarzine.

Conclusions: The presented data reinforce the need to improve the pediatricians' knowledge about the diagnosis and treatment of food allergy, in order to assure the use of appropriate diagnostic and treatment criteria.

Key-words: food hypersensitivity; knowledge; child; food additives; food; milk substitutes; egg white; peanuts; tartrazine.
1Professor titular e livre-docente da Escola Paulista de Medicina da Universidade Federal de São Paulo (Unifesp-EPM), São Paulo, SP, Brasil 2Doutor em Medicina pela Faculdade de Medicina da Universidade de São Paulo (FMUSP), médico da Unidade de Alergia e Imunologia do Instituto da Criança (ICr) do Hospital das Clínicas (HC) da FMUSP, São Paulo, SP, Brasil

${ }^{3}$ Mestre em Pediatria pela Universidade Federal do Rio Grande do Sul, Porto Alegre, RS, Brasil

4Presidente da Sociedade de Pediatria do Distrito Federal, Brasília, DF Brasil

${ }^{5}$ Professor adjunto de Pediatria da Universidade Federal de Pernambuco, Recife, PE, Brasil

${ }^{6}$ Professor assistente do Departamento de Pediatria da Universidade Federal do Rio de Janeiro, Rio de Janeiro, RJ, Brasil

'Professor adjunto do Departamento de Pediatria da Universidade Federal de Alagoas, Maceió, AL, Brasil
${ }^{8}$ Pesquisadora Associada e doutora em Medicina da Unifesp-EPM, São Paulo, SP, Brasil

${ }^{9}$ Mestre em Pediatria pela Unifesp-EPM e professora assistente de Pediatria da Faculdade de Medicina de Juazeiro do Norte, Juazeiro do Norte, CE, Brasil ${ }^{10}$ Livre-docente e professora adjunta da Universidade Federal do Estado do Rio de Janeiro, Rio de Janeiro, RJ, Brasil

Fonte financiadora: Nestlé (impressão e postagem dos questionários)

Endereço para correspondência:

Dirceu Solé

Rua Mirassol, 236, apto. 72 - Vila Mariana

CEP 04044-010 - São Paulo/SP

E-mail: dirceus@ajato.com.br

Recebido em: 22/3/2007

Aprovado em:15/8/2007 


\section{Introdução}

Há mais de uma década, a prevalência das doenças alérgicas tem sido avaliada por estudos epidemiológicos. $\mathrm{O}$ mais recente deles, o International Study of Asthma and Allergies in Childhood (ISAAC) se propôs determinar a prevalência de asma, rinite alérgica e eczema atópico em escolares empregando um instrumento padronizado, em várias partes do mundo ${ }^{(1)}$. Realizado em três fases sucessivas, o ISAAC permitiu verificar a real evolução dessas doenças após intervalo de tempo superior a seis anos e identificou o aumento da sua prevalência em algumas regiões ${ }^{(2)}$.

No Brasil, publicações recentes que comparam os resultados do ISAAC fase 1 e fase 3, com um hiato de sete anos entre ambos, mostram haver uma tendência à redução na prevalência de asma ativa, mas não de diagnóstico médico de asma entre os adolescentes avaliados em cinco capitais do país (Recife, Salvador, São Paulo, Curitiba e Porto Alegre $^{(3)}$. Resultados similares foram observados em relação à prevalência de rinite, rinoconjuntivite e de eczema atópico ${ }^{(3)}$. Apesar disso, os valores encontrados continuam entre os mais elevados da América Latina ${ }^{(2)}$.

Entretanto, em relação à alergia alimentar, esses dados são escassos e de obtenção muito difícil, uma vez que as manifestações clínicas são variadas e se confundem com diversas situações clínicas - em especial outras reações adversas a alimentos, que freqüentemente são consideradas como alergia alimentar ${ }^{(4,5)}$.

Até o presente momento, não há nenhum estudo de abrangência nacional ou internacional, como o ISAAC, que tenha sido realizado para avaliar a real dimensão da alergia alimentar em populações ao redor do mundo. Na maioria das vezes, os dados de prevalência ou incidência de alergia alimentar são obtidos com o estudo de pequenos grupos populacionais de risco ou não, com metodologia diagnóstica variável e, em geral, com resultados não extrapoláveis.

Um dos pontos diretamente relacionado à divergência nas taxas obtidas de alergia alimentar pelos diferentes estudos é o critério diagnóstico empregado. Tem sido observada oscilação na prevalência ou incidência da alergia alimentar em crianças do nascimento aos dois anos de vida desde $35 \%$, quando se baseia fundamentalmente em informações referidas pelos pais com relação aos seus filhos, a menos de $1 \%$, quando se utiliza o desencadeamento oral (aberto ou cego) para confirmação diagnóstica(6-8).

Em crianças holandesas de quatro a 15 anos de idade, avaliadas durante a consulta médica de rotina, a prevalência de reações adversas a alimentos foi de $7,2 \%$, segundo informações fornecidas por seus pais e/ou responsáveis. Os aditivos alimentares e o chocolate foram os alimentos mais apontados como responsáveis pelas manifestações clínicas. Além disso, os autores verificaram maior freqüência de faltas à escola, consumo de medicamentos, assim como de comprometimento do estado de saúde nas crianças com reações adversas a alimentos ${ }^{(8)}$. Com a certeza de que muitas vezes tais crianças são submetidas a dietas restritivas sem que qualquer avaliação diagnóstica tenha sido realizada, os autores recomendam que os pais desses pacientes sejam informados sobre os efeitos dessas restrições para a saúde de seus filhos e a indicação para buscar um diagnóstico mais preciso ${ }^{(8)}$.

É necessário ter em mente que as dietas restritivas muitas vezes podem atingir proporções graves. Liu $e t a^{(9)}$, em publicação recente, documentaram 12 crianças norteamericanas a eles referidas de centros terciários daquele país com quadro clínico de kwashiorkor. Em metade delas, este quadro de extrema desnutrição decorria de desvio deliberado da dieta carente em proteína, em pacientes com intolerância ao leite de vaca.

Segundo Keil ${ }^{(10)}$, os estudos epidemiológicos realizados com base nas informações obtidas dos pais e/ou responsáveis dos pacientes mostram, em geral, taxas superestimadas de alergia alimentar. De certo modo, cabe ao médico fornecer dados mais precisos a respeito desse possível diagnóstico, o qual muitas vezes exige a avaliação diagnóstica objetiva para obter dados mais fidedignos.

Neste ponto, não se pode esquecer que o nível de conhecimento do médico que atende a esses pacientes sobre as diferentes faces da alergia alimentar é muito importante. Estudo recente avaliou a conduta de médicos alergologistas, gastroenterologistas pediátricos, pediatras e médicos de saúde comunitária em relação à reatividade cruzada entre leite de vaca e soja. Avaliados por questionário escrito, verificou-se variabilidade grande à identificação e conduta tomada pelos médicos das diferentes especialidades. Segundo os autores, tais diferenças dependiam mais de conhecimentos gerais do que da própria experiência dos médicos avaliados. Ao final do estudo, os autores ressaltam a necessidade de implementação do conhecimento em alergia alimentar ${ }^{(11)}$.

Com esses fatos em mente e conhecedores da procura cada vez maior dos serviços de especialidade por pacientes com diagnóstico de alergia alimentar, sobretudo no primeiro ano de vida, e muitas vezes submetidos a dietas restritivas não corretamente embasadas por diagnóstico adequado de alergia alimentar ${ }^{(12)}$, foi intenção dos membros integrantes do Departamento de Alergia e Imunologia da Sociedade Brasileira de Pediatria (SBP) realizar esta pesquisa em amostra de pediatras, verificando o conhecimento deles sobre a alergia alimentar. 


\section{Métodos}

Questionário padrão elaborado pelos membros do Departamento Científico de Alergia e Imunologia (Quadro 1) foi distribuído a pediatras associados da SBP durante os meses de dezembro de 2005 e janeiro de 2006. Junto ao questionário, foi postada correspondência explicativa a respeito dos objetivos do estudo, deixando livre a necessidade de identificação do respondedor. $\mathrm{O}$ questionário foi postado com envelope selado e endereçado à sede da SBP, no Rio de Janeiro. Após serem respondidos, eles foram re-encaminhados à SBP e os dados obtidos foram, a seguir, transferidos a banco de dados por digitação dupla. Os dados foram analisados segundo a porcentagem de respostas afirmativas (SIM) às diferentes questões.

\section{Resultados}

Foram analisados 895 questionários respondidos pelos pediatras afiliados à SBP, com predomínio das regiões Sudeste $(61,6 \%)$ e Sul $(18 \%)$. Para cada item, o número de respostas foi variável. O tempo de prática pediátrica desses profissionais variou de um a 58 anos (mediana: 14 anos).

Em relação às manifestações clínicas mais apontadas, de modo isolado ou em associação, houve discreto predomínio das respiratórias $(48,5 \%, \mathrm{n}=434)$ em relação às cutâneas (45,5\%, $\mathrm{n}=407)$ e sistêmicas ( $43 \%, \mathrm{n}=385)$, sendo as alterações gastrintestinais referidas em menor freqüência $(32,4 \%, \mathrm{n}=289)$. Entre as manifestações respiratórias, predominou o chiado em $48,9 \%$, além da tosse $(21,8 \%)$, rinite $(15,6 \%)$ e otites de repetição $(13,7 \%)$. Entre as cutâneas, predominaram a urticária $(49,7 \%) \mathrm{e}$ a dermatite atópica $(43,7 \%)$. Edema de glote $(72,8 \%)$ e choque $(27,2 \%)$ foram as manifestações sistêmicas predominantes. As manifestações gastrointestinais incluíram: diarréia $(20,6 \%)$, vômitos (16,8\%), cólicas (16,2\%), diarréia com sangue (12,6\%), distensão abdominal $(11,2 \%)$ e refluxo gastroesofágico $(8,6 \%)$.

Os alimentos desencadeantes mais referidos como responsáveis por esses quadros $(\mathrm{n}=890)$ foram: leite de vaca $(98,9 \%$, $\mathrm{n}=880)$, clara de ovo $(58,7 \%, \mathrm{n}=522)$, amendoim $(50,9 \%$, $\mathrm{n}=453)$, peixes $(49,1 \%, \mathrm{n}=437)$, soja $(42,2 \%, \mathrm{n}=376)$, trigo $(38,3 \%, \mathrm{n}=341)$, chocolate $(6,7 \%, \mathrm{n}=60)$ e frutos do mar $(4,5 \%, \mathrm{n}=40)$. Os aditivos alimentares foram responsabilizados por $74,8 \%$ dos respondedores; entretanto, apenas $19,4 \%$ deles conheciam o código pelo qual a tartrazina é reconhecida.

Dentre os principais instrumentos para o diagnóstico da alergia alimentar $(\mathrm{n}=895)$, foram apontados: história clínica (97,5\%), dieta de exclusão (85\%), exame clínico (67,5\%), nível sérico de IgE específica $(59,2 \%)$, pesquisa de sangue oculto nas fezes $(36,3 \%)$, contagem de eosinófilos séricos $(30,8 \%)$, teste de provocação oral $(30,7 \%)$, teste cutâneo de leitura imediata $(22,5 \%)$ e a dosagem de alfa-1 antitripsina (10,2\%).

A conduta instituída ao diagnosticar alergia alimentar (n=895) foi: dieta de exclusão (75,7\%); anti-histamínicos H1 $(13,5 \%)$; adrenalina subcutânea $(7,1 \%)$; corticosteróide sistêmico $(2,4 \%)$; corticosteróide inalado (1\%) e antidiarréicos $(0,3 \%)$. Associada à dieta de exclusão, os cuidados adicionais tomados foram: exclusão do alimento suspeito e derivados (91,2\%); reposição mineral $(40,4 \%)$ e exclusão de alimentos com reatividade cruzada $(43,8 \%)$; embora muitos alimentos com reatividade cruzada não fossem considerados.

Em relação ao relato do desenvolvimento de tolerância ao leite de vaca, esta ocorreu em $70 \%$ dos pacientes, sendo $88 \%$ deles nos primeiros quatro anos, com tempo médio de manutenção de sua exclusão de 2,7 $\pm 1,5$ anos (variação: 0,1 a 12,5 anos) após o diagnóstico. Nos pacientes com alergia a peixes e crustáceos, $40,2 \%$ desenvolveram tolerância, no tempo médio de 4,4 42,5 anos (variação: 0,5 a 16 anos) após o diagnóstico, sendo $50 \%$ nos primeiros quatro anos.

Como substituto do leite de vaca $(\mathrm{n}=875)$ foram prescritos: proteína isolada de soja (65\%); extrato de soja ( $47,8 \%)$; hidrolisado protéico $(37,9 \%)$ e leite de cabra $(26,5 \%)$. Ter feito diagnóstico de alergia alimentar alguma vez foi referido por $96,2 \%$ dos pediatras entrevistados.

\section{Discussão}

$\mathrm{O}$ viés de informação é uma das limitações que apresentam estudos como este, sobretudo se considerarmos que os respondedores eram profissionais interessados em alergia alimentar. Apesar disso, e mesmo que a amostra avaliada tenha sido limitada, ela nos permitiu observar dados que certamente podem refletir o entendimento que o pediatra geral tem sobre a alergia alimentar.

Nesse estudo, as manifestações respiratórias foram as mais sugestivas para o diagnóstico da alergia alimentar aqui referida como chiados, tosse e rinite. Estes dados se contrapõem aos publicados, que apontam as dermatológicas como as mais freqüentes ${ }^{(4,5)}$. Além disso, várias são as causas de chiado e a alergia a alimentos é associada a manifestações respiratórias em uma pequena porcentagem de pacientes e raramente se apresenta como única manifestação clínica. Além disso, outro ponto controverso e que foi referido de forma significativa pelos pediatras que participaram deste estudo diz respeito à otite média como manifestação de alergia alimentar ${ }^{(13)}$. Até o presente momento, todos os estudos que tentaram 
relacionar a otite à alergia alimentar, sobretudo ao leite de vaca, apresentam resultados contraditórios ${ }^{(14-16)}$.

Entre as manifestações dermatológicas, verificamos ser a dermatite atópica apontada por um percentual significativo de pediatras. A dermatite atópica é uma doença inflamatória da pele que tem início, geralmente, após os primeiros meses de vida e em concomitância ao desmame e/ou à introdução de proteínas heterólogas na dieta do lactente. A associação entre dermatite atópica e alergia alimentar ainda permanece controversa; porém, quando se estuda a dermatite grave, vários autores consideram que até $30 \%$ dos casos podem ser desencadeados por alimentos, sobretudo a clara de ovo, entre outros ${ }^{(17,18)}$. Deve ser ressaltado que apenas a dieta de restrição pode não alterar o curso da dermatite atópica, o que faz com que muitos pesquisadores não a relacionem à alergia alimentar. Nestes casos, deve ser lembrado que a dermatite atópica é uma doença que inclui muitos fatores em sua fisiopatologia e, talvez, apenas uma interferência pode não mudar seu curso clínico ${ }^{(17,18)}$.

Estudos controlados e de base populacional apontam que a freqüência de reações mais graves não atingem $5 \%^{(19)}$ - valores muito inferiores aos aqui ao documentados. Entre as manifestações gastrintestinais, predominaram as $\operatorname{IgE}$ mediadas (diarréia, vômitos e cólicas).

Entre os principais possíveis agentes etiológicos, observou-se concordância com os dados obtidos por outros autores. O leite de vaca, geralmente a primeira proteína heteróloga a ser introduzida na dieta do lactente, foi o mais freqüentemente identificado, seguido pela clara de ovo e amendoim. Este dado se contrapõe ao relatado por autores americanos que apontam ser a prevalência de sensibilização ao amendoim significativamente mais elevada, possivelmente por sua introdução na dieta da criança em fase muito precoce da vida, fato que não ocorre entre nós ${ }^{(17-19)}$. Além disso, a identificação do chocolate como agente etiológico em $6,7 \%$ dos casos chama atenção, uma vez que este tem sido raramente referido como etiologia de alergia alimentar ${ }^{(19)}$.

Outro ponto que suscita muita confusão é a participação dos aditivos alimentares como agentes etiológicos da alergia alimentar. A freqüência com que isto ocorre é baixa. Além disso, quando presentes, as manifestações não são determinadas por mecanismos imunológicos, o que não as caracteriza como exemplos de alergia alimentar ${ }^{(4)}$. Este fato ressalta a supervalorização dada aos aditivos alimentares diante de pacientes com sintomas que lembram os de alergia alimentar. Apesar disso, constatou-se que, embora o relato entre a associação de alergia e aditivos alimentares fosse freqüente, pequena parcela dos entrevistados sabia o código de identificação da tartrazina, o principal corante envolvido nessas reações.

A não disponibilidade de método acessível para a confirmação diagnóstica da alergia alimentar torna a história clínica uma ferramenta de grande importância. Entretanto, o desconhecimento dos sintomas, assim como da sua evolução, tem favorecido o diagnóstico abusivo de alergia alimentar e, em consequência, há imposição de dietas de exclusão de forma inadequada.

A história natural da alergia alimentar é distinta na dependência do alimento envolvido ${ }^{(17-19)}$. Neste estudo, há relato de remissão de alergia a peixes e crustáceos, que, na maioria dos estudos, é apontada como persistente. Este fato possivelmente decorre de reações de intolerância que, em geral, são transitórias. Outro ponto que vale destacar diz respeito à melhora do quadro de alergia alimentar ao leite de vaca com o emprego de leite de cabra. Sabe-se que há homologia de $92 \%{ }^{(4,5)}$ entre as proteínas do leite de vaca e o de cabra, e que este não é a melhor alternativa terapêutica diante desses quadros. Mais uma vez, os dados fazem suspeitar de que o diagnóstico de alergia ao leite de vaca pode não ter sido adequado.

Em relação ao substituto do leite de vaca, nota-se que muitos pediatras ainda utilizam o extrato de soja, mesmo em crianças de baixa idade, provavelmente em decorrência de seu baixo custo em relação às fórmulas infantis. É importante ressaltar a necessidade de adequação deste substituto em relação à idade da criança e a indicação exclusiva do uso de fórmulas nutricionalmente adequadas para crianças menores de dois anos ${ }^{(19)}$. Medeiros $e t$ $a l$, ao avaliarem crianças com alergia à proteína do leite de vaca encaminhadas a serviço especializado, verificaram que, comparativamente à dieta de crianças normais de mesma faixa etária e sem restrições dietéticas, aquelas resultaram em redução na ingesta de energia, proteínas, lipídios, cálcio e fósforo - muitas vezes abaixo das referências de ingestão dietética ${ }^{(20,21)}$.

Embora os dados aqui relatados sejam provenientes de amostra reduzida dos pediatras do país, os resultados indicam a necessidade de um consenso sobre o diagnóstico e o tratamento da alergia alimentar, com o objetivo de garantir critérios mínimos e evitar tentativas dietéticas desastrosas às crianças, com interferência no ganho pôndero-estatural e convívio social a que toda criança tem direito.

\section{Agradecimentos}

Agradecemos à Nestlé - Nutrição Infantil, pelo apoio que viabilizou a confecção, postagem e distribuição dos questionários, assim como a sua digitação após reenvio. 
Quadro 1 - Questionário sobre inquérito nacional sobre alergia alimentar

\begin{tabular}{|c|c|c|}
\hline \multicolumn{3}{|c|}{ Inquérito nacional sobre alergia alimentar } \\
\hline Nome: ................................. & .... Cidade: & \\
\hline \multicolumn{3}{|c|}{ 1. Há quantos anos você atende crianças? .......... anos. } \\
\hline \multicolumn{3}{|c|}{ 2. Além da Pediatria Geral, você tem outra especialidade? } \\
\hline $\operatorname{Sim} \ldots$. & Não .... & Qual? . \\
\hline \multicolumn{3}{|l|}{ 3. Sua principal atuação é em: } \\
\hline Clínica/ambulatório & Privada .... & Pública \\
\hline Enfermaria & Privada .... & Pública \\
\hline PS/emergência & Privada .... & Pública \\
\hline
\end{tabular}

4. Assinale, por ordem de freqüência, as manifestações clínicas que fizeram você suspeitar de alergia alimentar ou que estavam presentes em seus pacientes?

Cutâneas: $\quad$ Angioedema .... Urticária .... Dermatite atópica ....

Gastrointestinais: Vômitos .... Diarréia .... Diarréia com sangue .... Cólicas ....

Distensão abdominal .... Edema de lábios e boca .... Refluxo ....

Respiratórias: $\quad$ Otites de repetição .... Tosse .... Broncoespasmo/chiado .... Rinite ....

Sistêmicas/anafilaxia: Edema de glote .... Choque anafilático ....

Outras:

5. Na sua experiência, quais os principais alimentos que você tem identificado como etiologia da alergia alimentar? Leite de vaca .... Clara de ovo .... Trigo .... Peixes .... Soja .... Amendoim ....

Outros:

6. Na sua experiência, os aditivos alimentares (corantes, conservantes, glutamato) provocaram algum evento adverso que se confundiu com alergia alimentar?

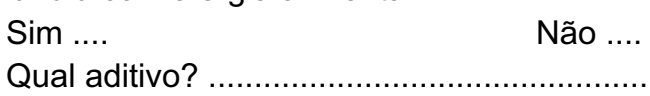

7. Você conhece códigos ou outras denominações da tartrazina?
Sim ....
Não ....
Desconheço esta substância ....

8. Como você faz o diagnóstico de alergia alimentar? Assinale uma ou mais alternativas

História .... Exame clínico .... Número de eosinófilos .... Sangue oculto fezes ....

Dosagem de alfa-1 tripsina das fezes .... Teste cutâneo puntura (prick) .... Rast - IgE específico ....

Dieta de exclusão .... Teste de provocação ....

9. A sua conduta inicial nesses casos tem sido (numere de 1 até 6):

Anti-histamínicos H1 .... Dieta de exclusão .... Corticosteróides (oral ou parenteral) ....

Antidiarréicos .... Corticosteróides inalados .... Adrenalina subcutânea ....

10. No caso de iniciar dieta de exclusão, que cuidados adicionais você toma?

Dieta de exclusão ao alimento suspeito e aos derivados ....

Excluir também os alimentos com reatividade cruzada ....

Avalia a necessidade de reposição mineral (pex. Cálcio) ....

11. Se você tem pacientes com alergia às proteínas do leite de vaca, em que porcentagem eles se tornaram tolerantes? E, em média, em quanto tempo?

$\%$ anos

12. Se você tem pacientes com alergia a peixes e crustáceos, em que porcentagem eles se tornaram tolerantes? $\mathrm{E}$, em média, em quanto tempo? $\%$ .anos

13. No caso de eliminação do leite de vaca e derivados, que substituto(s) usa?

Extrato hidrossolúvel de soja .... Leite de cabra .... Proteína isolada de soja .... Hidrolisado Protéico .... Outros. Citar:

14. Você já fez diagnóstico de alergia alimentar?

$\operatorname{Sim} . . .$.

Não .... 


\section{Referências bibliográficas}

1. Ellwood P, Asher MI, Beasley R, Clayton TO, Stewart AW; ISAAC Steering Committee. The International Study of Asthma and Allergies in Childhood (ISAAC): phase three rationale and methods. Int $\mathrm{J}$ Tuberc Lung Dis 2005;9:10-6

2. Asher MI, Montefort S, Björkstén B, Lai CK, Strachan D, Weiland SK et al. Worldwide time trends in the prevalence of symptoms of asthma, allergic rhinoconjunctivitis and eczema in childhood - ISAAC Phases One and Three repeat multicountry cross-sectional surveys. Lancet 2006;368:733-43.

3. Solé D, Melo KC, Camelo-Nunes IC, Freitas LS, Britto M, Rosário NA et al. Changes in the prevalence of asthma and allergic diseases among Brazilian schoolchildren (13-14 years old): comparison between ISAAC Phases One and Three. J Trop Pediatr 2007;53:13-21

4. Nowak-Wegrzyn A, Sampson HA. Adverse reactions to foods. Med Clin North Am 2006;90:97-127.

5. Sicherer SH, Sampson HA. Food allergy. J Allergy Clin Immunol 2006;117: S470-5.

6. Eggesbø M, Halvorsen R, Tambs K, Botten G. Prevalence of parentally perceived adverse reactions to food in young children. Pediatr Allergy Immunol 1999;10:122-32.

7. Jansen JJ, Kardinaal AF, Huijbers G, Vlieg-Boerstra BJ, Martens BP, Ockhuizen T. Prevalence of food allergy and intolerance in the adult Dutch population. $J$ Allergy Clin Immunol 1994;93:446-56.

8. Brugman E, Meulmeester JF, Spee-van der Wekke A, Beuker RJ, Radder JJ, Verloove-Vanhorick SP. Prevalence of self-reported food hypersensitivity among school children in The Netherlands. Eur J Clin Nutr 1998;52:577-81.

9. Liu T, Howard RM, Mancini AJ, Weston WL, Paller AS, Drolet BA et al. Kwashiorkor in the United States: fad diets, perceived and true milk allergy, and nutritional ignorance. Arch Dermatol 2001;137:630-6.

10. Keil T. Epidemiology of food allergy: what's new? A critical appraisal of recent population-based studies. Curr Opin Allergy Clin Immunol 2007;7:259-63.
11. Cohen A, Levy B, Leshno M, Katz Y. Food allergy--effect of physician attitude on the diagnosis and reported prevalence. Harefuah 2005;144:685-8.

12. Sinagra JL, Bordignon V, Ferraro C, Cristaudo A, Di Rocco M, Amorosi B et al. Unnecessary milk elimination diets in children with atopic dermatitis. Pediatr Dermatol 2007;24:1-6.

13. Lubianca Neto JF, Hemb L, Silva DB. Fatores de risco para otite média aguda recorrente: onde podemos intervir? - uma revisão sistemática da literatura. J Pediatr (Rio J) 2006;82:87-97.

14. Juntti $H$, Tikkanen $S$, Kokkonen J, Alho OP, Niinimäki A. Cow's milk allergy is associated with recurrent otitis media during childhood. Acta Otolaryngol 1999;119:867-73.

15. Hirata CH, Weckx LL, Solé D, Figueiredo CR. Serum levels of immunoglobulins in children with recurrent otitis media. J Investig Allergol Clin Immunol 1999;9:106-9.

16. Cantani A, Micera M. Natural history of cow's milk allergy. An eight-year followup study in 115 atopic children. Eur Rev Med Pharmacol Sci 2004;8:153-64

17. Sampson HA. The evaluation and management of food allergy in atopic dermatitis. Clin Dermatol 2003;21:183-92.

18. Leung DY, Nicklas RA, Li JT, Bernstein IL, Blessing-Moore J, Boguniewicz M et al. Disease management of atopic dermatitis: an updated practice parameter. Joint Task Force on Practice Parameters. Ann Allergy Asthma Immunol 2004;93(Suppl 2):S1-21.

19. American College of Allergy, Asthma \& Immunology. Food allergy: a practice parameter. Ann Allergy Asthma Immunol 2006;96(Suppl 2):S1-68.

20. National Research Council (NRC). Dietary Reference intakes for calcium phosphorus, magnesium, vitamin D, and fluoride. Washington (DC): National Academy Press; 1997.

21. National Research Council (NRC). Dietary reference intakes for energy, carbohydrate, fiber, fat, fatty acids, cholesterol, protein, and amino acids. Washington (DC): National Academy Press; 2002. 